\title{
Overlearning, extinction, and superstition effects on observing behavior in concept learning ${ }^{1}$
}

SVEN RYDBERG, Åbo Academy, Åbo, Finland, and PETER W. ARNBERG, ${ }^{2}$ University of Stockholm, Stockholm, Sweden

Observing in 36 concept-leaming $S s$ was recorded electrically. The 18 overlearning (O) Ss increased more in time (per cent) spent observing relevant stimuli, and then equaled 18 learming (L) Ss during extinction ( $E$ ) and prolonged extinction $(P$, $9+9$ Ss). Then, after the 3rd of 10 superstition trials lany response called "correct"), the resulting four groups of nine $S$ s consistently ranked $O E, O P, L E$, and $L P$ in previously relevant observing (mean per cent time).

Using direct measures (Rydberg, Kashdan, \& Trabasso, 1966) of overt observing, Rydberg (1969) and Rydberg \& Arnberg (1969) found that selective observing changed in human adults during concept learning. Mean per cent relevant touching tended to go up during overlearning, but there were occasional decreases and, in both studies, relevant touching leveled off around $80 \%{ }^{3}$ Overlearning has been used extensively as a tool for selective extinction and shaping of attentive behavior-without measures and control of the assumed attentional effects. We wanted to investigate further such observing effects under overlearning, extinction, and superstition conditions.

In extinction, the amount of previous overlearning had not had any notable effect on per cent time spent observing the previously relevant stimulus dimension (Rydberg \& Arnberg, 1969). We wondered what would happen in a superstition condition when the Ss previously have had different amounts of initial training and of extinction. We hypothesized that when Ss are "correct," regardless of their responses, Ss with overlearning should sooner or later have more of previously relevant observing; the amount of overlearning being constant, Ss with more prolonged extinction should sooner or later have less of previously relevant observing.

The Ss were to try to find out which one of four tactile stimulus dimensions was relevant and to press one of three response buttons for the corresponding intensity levels within that dimension. See Fig. 1. Each S went through: (1) discrimination pretraining, (2) concept-learning pretraining, (3) learning, (4) extinction, and (5) superstition conditions. When S pressed, a lamp at once indicated the correct response, except in (4) and (5). SUBJECTS

Thirty-six female students of educational psychology at the University of Stockholm served as Ss. APPARATUS

See Fig. 1. The experimental stimuli were contiguous to the upper surface of square blocks, $20 \times 37 \times 37 \mathrm{~mm}$, fastened in four holes, $10 \mathrm{~mm}$ from each other. Touch and classification responses, and their durations, were recorded. More details can be found in Rydberg et al (1966; changed circuitry has eliminated a temperature-stability requirement). ${ }^{4}$ STIMULI AND POSITIONS

Each object above represented one of three possible values in one of five stimulus dimensions: circle size (C), depth (D), height $(H)$, length (L), and roughness (R). We call the stimulus positions $a, b, c$, and $d$ (see Fig. 1). For stimulus details, see Rydberg \& Amberg (1969, Note 3). PROCEDURE AND DESIGN

In (1) and (2), the positions of the stimulus dimensions varied randomly from trial to trial, but thereafter, they were fixed. Thus, in the three main experiments, $S$ got to know which dimension-but not which value-he would find in each one of the four positions.

Instructions and pretraining taught $S$ (1) about the character of the stimuli, (2) to discriminate, (3) that only one dimension was relevant, (4) that in the relevant dimension, the least intensive stimulus was the cue for the leftmost response button, No. 1, medium for No. 2 , and high for No. 3, and (5) to stop feeling the stimuli before responding.

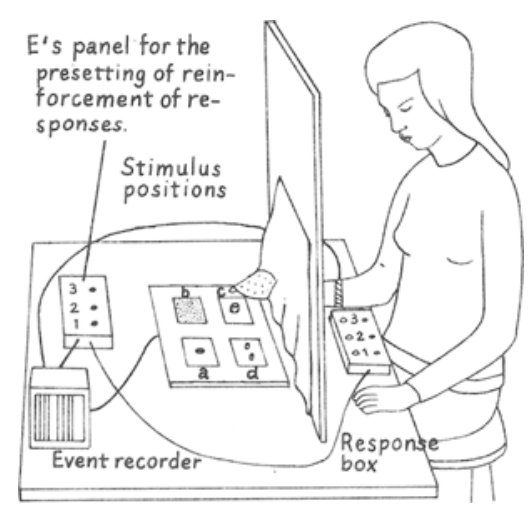

Fig. 1. Schematic apparatus description. In particular, stimuli are enlarged.
(1) Discrimination Pretraining

The E let $S$ see and touch the stimulus objects. E labeled the five dimensions and showed the four positions. Then $E$ seated $S$, as in Fig. 1, and let $S$ feel the three values of one dimension at a time, label it, and then label the values. Then $E$ gave one value at a time and instructed $S$ which button to press for each intensity value. $E$ successively excluded dimensions where $S$ had made three correct presses in a row.

(2) Concept-Learning Pretraining

The $E$ fastened blocks from four different dimensions. $\mathrm{E}$ always included $\mathrm{H}$ and told $S$ it was relevant for $t$ ' 'stton pressing. In three trials, E told s which button to press to be correct. E then gave more trials, now without help, until $S$ got five correct trials in a row.

\section{(3) Learning}

Dimension $\mathrm{H}$ was omitted. C was made relevant. $L, C, R$, and $D$ always were in Positions a, b, c, and d, respectively. (In Rydberg \& Arnberg, 1969, Dimension C and Position $b$ had been touched least.) The same instructions were given as in (2). Half of che Ss were run to a criterion of 12 (overlearn. $7 g$ ) and the others to 5 correct trials in a row.

(4-5) Extinction and Superstition

As above, but $\mathrm{E}$ showed $\mathrm{S}$ that all lamps would light up in case of a correct response. E surreptitiously manipulated the lamp circuits and signaled "wrong" in five trials (4) and "correct" in the final 10 trials for half of the previous "overlearners" and half of the others (5); the other group halves got 12 "wrong" and 10 "correct." Now $D, R, C$, and $L$ were in Positions a-d.

This gives four subgroups called: overlearning/prolonged extinction (OP), overlearning/extinction (OE), learning/prolonged extinction (LP), and learning/extinction (LE).

\section{RESULTS AND DISCUSSION}

Throughout, no $S$ failed. Two Ss reached criterion without any error. The slowest $S$ had 15 precriterion trials. The median was 2.4 trials.

As in the studies by Rydberg (1969) and Rydberg \& Amberg (1969), relative relevant touching increased before and during the criterion period. As in Rydberg \& Arnberg (1969), the increase before the criterion period took place in the trial of last error. This supports the explanation by Rydberg \& Arnberg (1969) that the gradual precriterion increase in Rydberg (1969) was an artifact from uncertainty in Ss about stimulus-response associations. After the criterion period (Fig. 2a), relative relevant touching fell to chance level when extinction began (Fig. 2b).

In the subsequent superstition condition, our hypotheses in the introduction above implied that the 


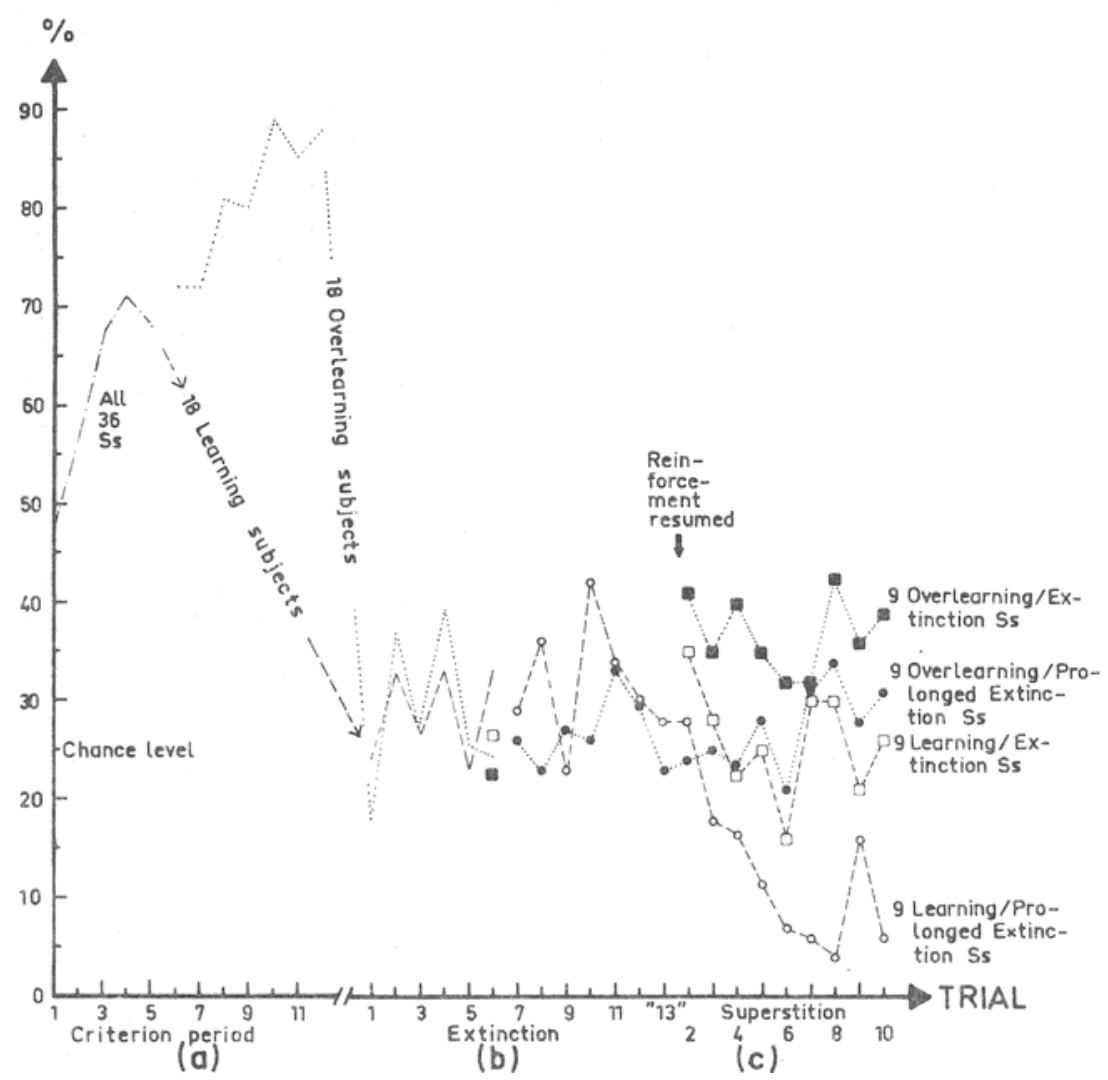

experimental group means should rank, in per cent previously relevant touching, as they indeed do in the last seven trials (see Fig. 2c). If the variation within and between trials were random, the probability of the run of consistently ordered means for $\mathrm{OE}, \mathrm{OP}, \mathrm{LE}$, and $\mathrm{LP}$ would be less than $1 / 10^{4}$ for the last three trials and less than $1 / 10^{10}$ for the last seven trials. ordinary learners and overlearners.
Fig. 2. Mean per cent touching time on the dimension that was relevant during learning for (a) criterion period, (b) extinction, and (c) superstition condition. (The last extinction data for each subgroup are based on touching in Superstition Trial 1.)

We tentatively conclude (1) that previous overlearning strengthens a tendency to revert to previously relevant stimuli under superstition conditions after extinction, and (2) that extinction weakens it. Observing behavior under superstition conditions after extinction might be related to personality differences, e.g., in flexibility-rigidity. Persons with higher rigidity scores would be expected to revert more to observing previously relevant stimuli.

\section{REFERENCES}

RYDBERG, S. Concept learning: Measured selective attending changes. Psychonomic Science, 1969, 16, 293-295.

RYDBERG, S., \& ARNBERG, P. W. Selective attending changes during concept learning, extinction, and superstition. Psychonomic Science, $1969,17,98-99$.

RYDBERG, S., KASHDAN, R., \& TRABASSO, $T$. Recording of tactile observing responses for the study of selective attention. Psychonomic Science, 1966, 6, 197-198.

\section{NOTES}

It is, of course, very possible that this trend of group means could be broken under even more prolonged superstition conditions. This nearly happened in Superstition Trial 7 (Fig. 2c). The superstition period in Rydberg \& Amberg (1969) of only five trials was probably too short to give a clear-cut difference between
1. Support: Swedish Social Science Research Council grants.

2. We thank Vera Jepson and Karin Lundström for assistance with data analysis, and Dr.T. Trabasso, UCLA, for stimulus blocks with scaling data.

3. The chance levels differed $67 \%$ and $25 \%$, respectively.

4. To facilitate evaluation, all three stimulus intensity levels were used in every trial (e.g., 1, 2, 3,3 ). 